\section{RECENT ADVANCES IN PHYSICS}

Reports on Progress in Physics

Vol. 12 (1948-49). Pp. iii +382. (London: Physical Society, 1949.) 42s. net; to Fellows, 25s. net.

$\mathrm{I}^{\mathrm{N}}$ recent years almost every branch of physics has been expanded considerably. Furthermore, the volume of scientific publications has increased to such a large extent that even the specialist in the narrowest field finds it difficult to keep in touch with the rapid developments in his own subject. To follow, in addition, major advances in other important branches of physics is practically impossible. The need, therefore, for the publication at frequent intervals of specialist surveys and reviews of the different fields of physics, such as those provided by "Reports on Progress in Physics", could not be greater. The welcome return, with the publication of Vol. 11 in 1948, the present work in 1949 and the appearance of Vol. 13 in the very near future, to the annual series from the temporary two-year volumes is not only a matter for congratulation but also indicates that the Physical Society is fully alive to the growth of physics and the needs of physicists.

As in previous volumes, the fourteen articles contained in Vol. I2 are authoritative and masterly contributions written by well-known experts in their ruspective subjects. It is a moot point, however, whether the author of a report should consider his reader to be a specialist worker in that particular field or a trained physicist seeking an insight into, and understanding of, the recent advances in other branches of physics than that peculiarly his own. Both kinds of reports would be equally useful. It is impossible, however, to tell from the title alone into which of these two categories any one of the reports of Vol. 12 falls. In some, as a welcome innovation, a contents list has been provided, and in a few, also, an abstrect, so that an idea of the scope of a particular report can be obtained at a glance. But it is a pity that the editor has not insisted on uniformity in this connexion. The provision of an abstract is most helpful, if not essential, and the insertion, at the editorial stage, of a contents list for each report is surely a simple matter.

As usual the contributions cover advances in both the experimental and theoretical fields. On the experimental side there are articles on mass spectrometry; nuclear paramagnetism ; phosphors and phosphores. cence ; nuclear experiments with high-voltage X-rays, in which nuclear physical experiments carried out with betatrons and synchrotrons are described; linear accelerators in which both electron accelerators and the standing-wave proton accelerator are dealt with; and on low-temperature physics in which $\mathbf{K}$. Mendlessohn very rightly restricts his attention to the recent advances in technique and to the two physical phenomena peculiar to low temperatures, namely, superconductivity and superfluidity.

On the theoretical side the volume contains several reports of interest, including one on the theory of oxidation by N. Cabrera and N. F. Mott, and one on collisions between atoms and molecules at ordinary temperatures by $\mathrm{H}$. S. W. Massey. In addition, there are two rather longer articles which are more of the class of monographs than raports. The first, by E. Orowan, is on the fracture and strength of solids. In some forty-five pages, after outlining the main types of fracture, a detailed discussion is given of ultimate stress and necking; brittle fracture, ductile fractures and notch brittleness; fatigue; intercrystalline fractures and fracture by molecular sliding. The second, which is even longer, is by J. de Boer, on molecular distribution and the equation of state of gases. Both the kinetic method and the method of statistical mechanics are clearly explained. A section of a more practical nature follows, and, in order not to lengthen the treatment too much, only the applica. tion to the calculation of the distribution-function and the caloric and thermal equation of state of gases, and the properties of systems in equilibrium are discussed.

Three other reports call for special comment. The first, "Spontaneous Fluctuations", by D. K. C. MacDonald, will be of interest both to pure and electronic physicists. It is an account of 'noise' in electrical circuits, in valves and in semi-conductors. Particular attention is paid to the most recent developments. The second, by G. O. Jones, on viscosity and related properties in glass, which was originally presented before the Physical Society as a lecture survey on February 15, 1946, should appeal to the general reader as well as the glass technologist. The flow properties of glass are of obvious practical importance, but, since glass can be regarded as $\mathrm{c}$ liquid even at temperatures where it most closely resembles a solid, it is also of considerable theoretical interest. The structure and general properties of glass are briefly considered, and then the significance of phenomena in the transformation region. Finally, the report by M. Ross and J. S. Story on slow. neutron absorption cross-sections of the elements is nothing more than a list of published declassified numerical values, and, though of considerable value, seems out of place in "Progress Reports".

All the excellent features present in previous issues appear in Vol. 12, including the extensive bibliographies, the author index, the clear linediagrams and beautifully reproduced photographs. The paper, printing and binding are of the highest standard and similar to those of the Physical Society's Proceedings and its other publications. The "Reports" are an essential reference work, and no library or active physicist can afford to be without a copy.

S. WeINTROUB

\section{AN ALPHABETICAL GUIDE TO ATOMIC ENERGY}

Pocket Encyclopedia of Atomic Energy

Edited by Frank Gaynor. Pp. 204. (New York : Philosophical Library, Inc., 1950.) 7.50 dollars

HIS book consists of about two thousand definitions or short explanations of terms which are commonly used in discussing atomic energy and nuclear physics. The entries vary in length from a few words to a few pages, and are supplemented by about thirty-five diagrams and tables. There are also brief descriptions of some of the more important nuclear research laboratories and biographical sketches of a few well-known men of science. German equivalents are given for the majority of the terms which have been included.

The intrusion of highly specialized expressions from nuclear physics and engineering into wide fields of politics, administration and technology fully justifies the preparation of such a glossary. Many 